\title{
Relationship between microstate number and available entransy
}

\author{
CHENG XueTao \& LIANG XinGang \\ Key Laboratory for Thermal Science and Power Engineering of Ministry of Education, Department of Engineering Mechanics, Tsinghua \\ University, Beijing 100084, China
}

Received January 14, 2012; accepted February 24, 2012

\begin{abstract}
Based on the relationship between entransy and microstate number, we discuss the variations of the available transport entransy, the unavailable transport entransy, the available conversion entransy and the unavailable conversion entransy with the microstate number. We focus on physical processes in which heat is used for heating/cooling or doing work. When heat is transported for heating or cooling, the available transport entransy increases if the increase in microstate number is due to the increase in internal energy of the system, and decreases if the increase in microstate number is due to spontaneous heat transfer. When heat is used to do work, both the available conversion entransy and the unavailable conversion entransy increase if the increase in microstate number relates to the growth in internal energy of the system. The available conversion entransy decreases and the unavailable conversion entransy increases if the increase in microstate number results from spontaneous heat transfer.
\end{abstract}

microstate number, entransy, available entransy, unavailable entransy

Citation: Cheng X T, Liang X G. Relationship between microstate number and available entransy. Chin Sci Bull, 2012, 57: 3244-3250, doi: 10.1007/s11434-012$5152-9$

Heat transport is one of the most universal phenomena in nature. There are two main applications of heat. One is to transport heat for doing work, such as various heat engines, while the other is to transport heat for heating or cooling, such as various heat exchangers. The first involves heatwork conversion, while the other does not. Based on this difference, researchers have developed different physical quantities and principles.

For thermodynamic processes in which heat is transported for doing work, Clausius introduced an important state function, entropy, when he investigated the Carnot cycle in 1854 [1,2]:

$$
\mathrm{d} S=\left(\frac{\delta Q}{T}\right)_{\mathrm{rev}},
$$

where $\mathrm{d} S$ is the variation of entropy, $\delta Q$ is the heat exchange between the system and the environment, and $T$ is the temperature of the heat source, which is also the environmental temperature for reversible processes. The subscript rev indicates that the process is an ideal reversible

*Corresponding author (email: liangxg@ $@$ tsinghua.edu.cn) process. It is found that entropy can represent the energy grade [1,2]. Greater entropy generation during one physical process means a greater decrease of the energy grade, and more loss of the ability of doing work. Therefore, the concept of entropy generation can be used to optimize heatwork conversion to reduce the loss of the ability of doing work and increase the energy utilization efficiency [3].

For processes in which heat is transported for heating or cooling, Guo et al. [4] proposed a physical quantity, entransy, based on the analogy between heat conduction and electrical conduction. For an equilibrium system, the macroscopic definition of entransy is [4]

$$
G=\frac{1}{2} U T,
$$

where $U$ is the internal energy and $T$ is the temperature of the system. The entransy in an object represents the ability of releasing heat. It relates not only to the temperature, but also to the thermal energy of the system. The essence of the entransy of an object is the 'potential energy' of heat, similar to the potential energy of water in a reservoir. When heat is transported from a higher-temperature body to a low- 
er-temperature one, energy is conserved, but the transport ability of the heat decreases because entransy is always dissipated in the process [4]. Based on the concept of entransy dissipation, Guo et al. [4] developed the extremum entransy dissipation principle, defined the entransy-dissipation-based thermal resistance, and proposed the minimum thermal resistance principle. It was found that the principles of the entransy theory are appropriate to the optimization of heat conduction [5-13], heat convection [14-17], thermal radiation, design of heat exchangers [21-27] and phase change heat transfer processes [28].

Eqs. (1) and (2) express the macroscopic definitions of entropy and entransy. From the microscopic view, researchers have set up relationships between these concepts and the microstate number $\Omega$ that measure the degree of disorder of the system [1,2,29,30]. A bigger microstate number means a larger degree of disorder. Boltzmann established the connection between the entropy of a quasi-independent particle system and its microstate number $\Omega$ in 1877:

$$
S \propto \ln \Omega .
$$

In 1900, Planck introduced a proportional coefficient, and rewrote eq. (3) as [1,2,29]

$$
S=k \ln \Omega,
$$

where $k$ is the Boltzmann constant. This is the famous Boltzmann entropy equation, which is a bridge between entropy and microscopic particle theory, and makes us know the concept of entropy more deeply.

Cheng et al. [30] derived the relation between entransy and the microstate number for a monatomic ideal gas system. It is

$$
G=\kappa \Omega^{4 /(3 N)},
$$

where the proportional coefficient $\kappa$ is

$$
\kappa=\beta\left(N^{7 / 3} / m^{2} V^{4 / 3}\right),
$$

where $\beta$ is a constant whose value is $9.463 \times 10^{-114} \mathrm{~J}^{3} \mathrm{~s}^{4} \mathrm{~K}, N$ is the number of molecules, $m$ is the molecular mass, and $V$ is the volume of the system. We see that $\kappa$ is constant for a system with a fixed number of molecules, molecular mass and volume. For instance, when the volume of $1 \mathrm{~mol}$ helium gas is $1 \times 10^{-3} \mathrm{~m}^{3}$, the value of $\kappa$ is $0.0656 \mathrm{~J} \mathrm{~K}$.

Eqs. (4) and (5) are the expressions for the microscopic entropy and microscopic entransy of a system, and are the connections between the macroscopic quantities and the microstate number. However, for a practical heat-work conversion or a practical heat transfer process, it is worth further investigating the relationship between the microstate number and the ability of doing work or transporting heat for the thermal energy in a system. Based on the concept of entransy, $\mathrm{Wu}$ [31] discussed heat transfer and heat-work conversion, defined transport entransy as the ability of transporting heat for heating or cooling objects, and defined conversion entransy as the ability of transporting heat for doing work. Based on the entransy classification of $\mathrm{Wu}$ [31], we now set up and discuss the relationship between the microstate number and the ability of doing work or transporting heat for a system.

\section{Relationship between microstate number and available transport entransy}

Let us discuss the case in which heat is transported for heating or cooling objects. As shown in Figure 1, the internal energy of the system is $U$. When heat is transferred between the system and other objects, the entransy of the system is reduced. The decrease is named the available transport entransy, whose differential expression is [32]

$$
\mathrm{d} G_{\mathrm{a}-\operatorname{tran}}=T \mathrm{~d} U,
$$

where $\mathrm{d} U$ is the change of the internal energy, and $T$ is the temperature of the system.

There will be no spontaneous heat transfer if the system in Figure 1 is at equilibrium. We assume that the initial temperature of the system is $T_{1}$, and that the heat is transported between the system and the cold source with constant temperature $T_{0}$. According to the second law of thermodynamics, the lowest possible temperature of the system is $T_{0}$. Therefore, the available transport entransy of the system is

$$
G_{\mathrm{a}-\operatorname{tran}}=\int_{0}^{T_{1}} \mathrm{~d} G_{\mathrm{a}-\operatorname{tran}}=G_{1}-G_{0},
$$

where $G_{1}$ is the entransy of the system at temperature $T_{1}$, while $G_{0}$ is that at temperature $T_{0}$. The remaining entransy after excluding the available transport entransy from the system is called the unavailable transport entransy, which is

$$
G_{\text {ua-tran }}=G_{1}-G_{\text {a-tran }}=G_{0} .
$$

Based on eq. (5), when the volume, number of molecules and molecular mass are fixed, the relation between the available transport entransy and the microstate number can be expressed as

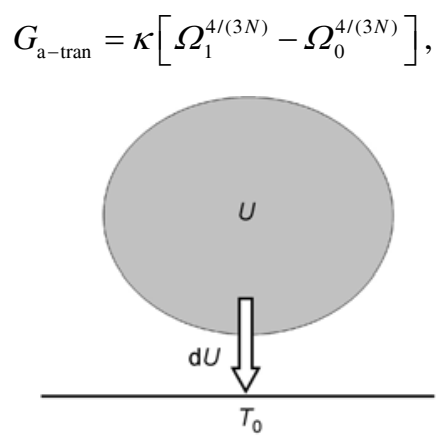

Figure 1 Sketch of heat transfer. 
where $\Omega_{1}$ is the microstate number of the system at temperature $T_{1}$, and $\Omega_{0}$ is that at temperature $T_{0}$. The relation between the unavailable transport entransy and the microstate number is

$$
G_{\text {ua-tran }}=\kappa \Omega_{0}^{4 /(3 N)} .
$$

The available transport entransy is determined by the microstate numbers at $T_{1}$ and $T_{0}$ according to eqs. (10) and (11), while the unavailable transport entransy is only determined by the microstate number of the system at $T_{0}$. The relationship between the temperature and the microstate number is [30]

$$
T=\alpha \Omega^{2 /(3 N)},
$$

where $\alpha$ is constant for a system with fixed volume, number of molecules and molecular mass. Bigger $\Omega_{0}$ means a higher $T_{0}$, which in turn makes the unavailable transport entransy of the system become larger and the available transport entransy smaller for given $\Omega_{1}$. On the other hand, raising the initial temperature of the system by increasing its initial internal energy of the system will makes $\Omega_{1}$ increase, thus the available transport entransy will be increased for prescribed $\Omega_{0}$.

Let us further consider an isolated system whose state is not in equilibrium at initial time. In Figure 2, the system is composed of two subsystems with the same numbers of molecules $N_{\mathrm{s}}$, the same molecular masses $m$, the same volumes $V_{\mathrm{s}}$, but different initial temperatures. Therefore, the proportional coefficients of entransy $\kappa_{\mathrm{s}}$ are the same for both subsystems. Assume that there is a plate with ideal heat insulation between the subsystems, and that there is no energy transfer between the subsystems before the plate is removed.

When the ideal plate is not removed, the heat transfer between the system and the cold source with temperature $T_{0}$ can be treated as separate heat transfer processes between the two subsystems and the cold source. According to eq. (10), the available transport entransies of the subsystems are

$$
G_{\mathrm{a}-\text { tran }-\mathrm{s} 1}=\kappa_{\mathrm{s}}\left[\Omega_{\mathrm{sl}}^{4 /(3 N)}-\Omega_{\mathrm{s} 1-0}^{3 /\left(3 N_{\mathrm{s}}\right)}\right],
$$

and

$$
G_{\mathrm{a}-\operatorname{tran1}-\mathrm{s} 2}=\kappa_{\mathrm{s}}\left[\Omega_{\mathrm{s} 2}^{4 /\left(3 N_{\mathrm{s}}\right)}-\Omega_{\mathrm{s} 2-0}^{4 /\left(3 N_{\mathrm{s}}\right)}\right],
$$

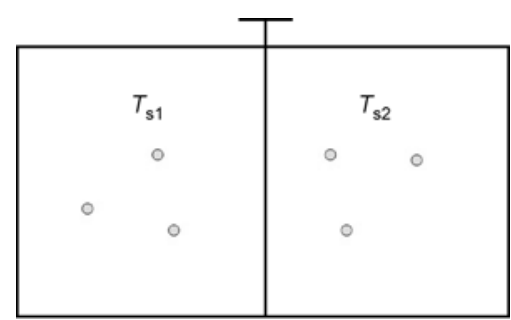

Figure 2 A non-equilibrium isolated system composed of two subsystems. where $\Omega_{\mathrm{s} 1}$ and $\Omega_{\mathrm{s} 2}$ are the initial microstate numbers, and $\Omega_{\mathrm{s} 1-0}$ and $\Omega_{\mathrm{s} 2-0}$ are the microstate numbers of the subsystems at temperature $T_{0}$. Because the subsystems have the same $N_{\mathrm{s}}$, $m$ and $V_{\mathrm{s}}, \Omega_{\mathrm{s} 1-0}=\Omega_{\mathrm{s} 2-0}$ based on eq. (12), and we use $\Omega_{\mathrm{s} 0}$ to express them. Therefore, the total available transport entransy of the system is

$$
\begin{aligned}
G_{\mathrm{a}-\text { tran }} & =G_{\mathrm{a}-\text { tran }-\mathrm{s} 1}+G_{\mathrm{a}-\operatorname{tran} 1-\mathrm{s} 2} \\
& =\kappa_{\mathrm{s}}\left[\Omega_{\mathrm{s} 1}^{4 /\left(3 N_{\mathrm{s}}\right)}+\Omega_{\mathrm{s} 2}^{4 /\left(3 N_{\mathrm{s}}\right)}\right]-2 \kappa_{\mathrm{s}} \Omega_{\mathrm{s} 0}^{4 / 3\left(N_{\mathrm{s}}\right)} .
\end{aligned}
$$

Correspondingly, the unavailable transport entransy is

$$
G_{\text {ua-tran }}=2 \kappa_{\mathrm{s}} \Omega_{\mathrm{s} 0}^{4 /\left(3 N_{\mathrm{s}}\right)} .
$$

According to eq. (15), the available transport entransy of the whole system will increase if either of the subsystems' initial microstate number is raised by energy from outside.

On the other hand, let's remove the ideal plate and make the two subsystems reach equilibrium by spontaneous heat transfer before heat transfer occurs between the system and the cold source. In this case, the available transport entransy of the whole system is

$$
G_{\mathrm{a}-\operatorname{tran}-\mathrm{eq}}=\kappa\left[\Omega_{\mathrm{eq}}^{4 /(3 N)}-\Omega_{0}^{4 /(3 N)}\right],
$$

where $\Omega_{\mathrm{eq}}$ is the microstate number of the whole system at equilibrium, and $\Omega_{0}$ is the microstate number of the whole system at $T_{0}$. Correspondingly, the unavailable transport entransy is

$$
G_{\text {ua-tran-eq }}=\kappa \Omega_{0}^{4 /(3 N)} .
$$

For the system in Figure 2, the microstate number of the whole system equals the product of the microstate numbers of the subsystems. Therefore, at the initial time,

$$
\Omega_{1}=\Omega_{\mathrm{s} 1} \Omega_{\mathrm{s} 2} .
$$

When the temperature of the system reaches that of the cold source,

$$
\Omega_{0}=\Omega_{\mathrm{s} 1-0} \Omega_{\mathrm{s} 2-0}=\Omega_{\mathrm{s} 0}^{2} .
$$

Considering eq. (6), we get

$$
\kappa=2 \kappa_{\mathrm{s}} .
$$

Combining eqs. (16), (18), (20) and (21) with $N=2 N_{\mathrm{s}}$ leads to

$$
G_{\text {ua-tran-eq }}=G_{\text {ua-tran }} .
$$

According to eq. (22), the unavailable transport entransy will not change no matter whether the system in Figure 2 is at equilibrium or not. However, the entransy of the system will decrease during the thermal equilibrium process [30]. Accordingly,

$$
\kappa_{\mathrm{s}}\left[\Omega_{\mathrm{s} 1}^{4 /\left(3 N_{\mathrm{s}}\right)}+\Omega_{\mathrm{s} 2}^{4 / 3\left(N_{\mathrm{s}}\right)}\right]>\kappa \Omega_{\mathrm{eq}}^{4 /(3 N)} .
$$


Therefore, combining eqs. (15), (17), (22) and (23) gives

$$
G_{\text {a-tran }}>G_{\text {a-tran-eq }} \text {. }
$$

According to the discussion in [30],

$$
\Omega_{1}<\Omega_{\text {eq }}
$$

for the initial and thermal equilibrium states. We see that the available transport entransy of the isolated system in Figure 2 will decrease with increasing microstate number.

The above discussions tell us that the unavailable transport entransy is determined only by the microstate number $\Omega_{0}$ at the cold source temperature $T_{0}$ when heat transfer occurs between the system and the cold source. Bigger $\Omega_{0}$ leads to bigger the unavailable transport entransy and smaller the available transport entransy. The available transport entransy of the system relates not only to $\Omega_{0}$ but also the initial microstate number $\Omega_{1}$. It increases with increasing $\Omega_{1}$ if $\Omega_{1}$ is raised by the energy transported into the system. If the increase of $\Omega_{1}$ results from the spontaneous heat transfer in the system, the available transport entransy of the system decreases with increasing $\Omega_{1}$.

\section{Relationship between microstate number and available conversion entransy}

Part of the entransy disappears in the heat-work conversion when thermal energy is used to do work. This part of the entransy is called the available conversion entransy. We first discuss the case in which there is no spontaneous heat transfer in a system. As shown in Figure 3, a Carnot engine works between a system with internal energy $U$ and a cold source with temperature $T_{0}$, and outputs mechanical work. The system itself does not directly involve work. The first law of thermodynamics gives

$$
\delta Q=\mathrm{d} U
$$

where $\delta Q$ is the heat transported into the heat engine from the system, and $\mathrm{d} U$ is the change of the system internal energy.

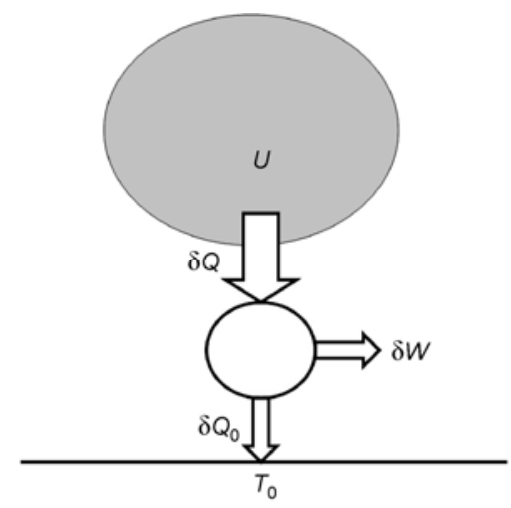

Figure 3 Sketch of the Carnot cycle between the system and the cold source.
The entransy absorbed by the Carnot engine from the system is

$$
\mathrm{d} G_{\text {in }}=T \delta Q=T \mathrm{~d} U,
$$

where $T$ is the temperature of the system. The entransy released to the cold source from the heat engine is

$$
\mathrm{d} G_{\text {out }-0}=T_{0} \delta Q_{0}=T_{0} \mathrm{~d} U_{0},
$$

where $\delta Q_{0}$ is the heat released to the cold source from the heat engine, and $\mathrm{d} U_{0}$ is the change of the internal energy of the cold source. The difference between eqs. (27) and (28) is the part of the entransy $\mathrm{d} G_{\text {in }}$ absorbed from the system that is consumed in the heat-work conversion in the Carnot engine. Therefore, the differential expression of the available conversion entransy can be defined as

$$
\mathrm{d} G_{\text {a-conv }}=T \mathrm{~d} U-T_{0} \mathrm{~d} U_{0} .
$$

According to the Carnot cycle,

$$
\frac{\mathrm{d} U}{T}=\frac{\mathrm{d} U_{0}}{T_{0}}
$$

Substituting eq. (30) into eq. (29) yields

$$
\mathrm{d} G_{\mathrm{a}-\mathrm{conv}}=\left(T-\frac{T_{0}^{2}}{T}\right) \mathrm{d} U .
$$

The temperature of the system must be higher than that of the cold source for the Carnot engine to absorb heat from the system and work between the system and the cold source. Therefore, the available conversion entransy of the system is

$$
G_{\text {a-conv }}=\int_{T_{0}}^{T_{1}} \mathrm{~d} G_{\text {a-conv }}=\int_{T_{0}}^{T_{1}}\left(T-\frac{T_{0}^{2}}{T}\right) \mathrm{d} U,
$$

where $T_{1}$ is the initial temperature of the system. Based on eq. (2) and the relationship between the internal energy and the temperature,

$$
G_{\text {a-conv }}=G_{1}-G_{0}-2 G_{0} \ln \frac{T_{1}}{T_{0}} .
$$

Substituting eqs. (5) and (12) into eq. (33) gives

$$
G_{\mathrm{a}-\mathrm{conv}}=\kappa \Omega_{1}^{4 /(3 N)}-\kappa \Omega_{0}^{4 /(3 N)}-\frac{4 \kappa}{3 N} \Omega_{0}^{4 /(3 N)} \ln \frac{\Omega_{1}}{\Omega_{0}} .
$$

Correspondingly, the unavailable conversion entransy is

$$
G_{\text {ua }- \text { conv }}=\kappa \Omega_{0}^{4 /(3 N)}+\frac{4 \kappa}{3 N} \Omega_{0}^{4 /(3 N)} \ln \frac{\Omega_{1}}{\Omega_{0}} .
$$

To discuss the relationship between the initial microstate number of the system $\Omega_{1}$ and the available conversion entransy, we assume that the microstate number of the system at the cold source temperature, $\Omega_{0}$, does not change. Then, the partial derivative of eq. (34) is 


$$
\frac{\partial G_{\mathrm{a}-\mathrm{conv}}}{\partial \Omega_{1}}=\frac{4 \kappa}{3 N} \Omega_{1}^{-1}\left[\Omega_{1}^{4 /(3 N)}-\Omega_{0}^{4(3 N)}\right] .
$$

The temperature of the system must be higher than that of the cold source, so $\Omega_{1}$ is bigger than $\Omega_{0}$ based on eq. (12). Then, we have

$$
\frac{\partial G_{\mathrm{a}-\mathrm{conv}}}{\partial \Omega_{1}}>0 .
$$

For the system in Figure 3, the available conversion entransy of the system will increase when $\Omega_{1}$ is increased by energy input from outside.

For the unavailable conversion entransy of the system, the partial derivative of eq. (35) gives

$$
\frac{\partial G_{\text {ua-conv }}}{\partial \Omega_{1}}=\frac{4 \kappa}{3 N} \Omega_{0}^{4 /(3 N)} \Omega_{1}^{-1}>0 .
$$

The unavailable conversion entransy of the system also increases with the growth of $\Omega_{1}$ that is due to energy input into the system.

We next discuss the influence of $\Omega_{0}$ on the available conversion entransy and the unavailable conversion entransy of the system. Assuming that $\Omega_{1}$ does not change, the partial derivative of eq. (36) gives

$$
\frac{\partial G_{\mathrm{a}-\mathrm{conv}}}{\partial \Omega_{0}}=-\frac{16 \kappa}{9 N^{2}} \Omega_{0}^{4 /(3 N)-1} \ln \frac{\Omega_{1}}{\Omega_{0}}<0 .
$$

It is clear that the available conversion entransy of the system will decrease with increasing $T_{0}$ and $\Omega_{0}$. The unavailable conversion entransy behaves in an opposite manner. It can be proved that

$$
\frac{\partial G_{\mathrm{ua}-\mathrm{conv}}}{\partial \Omega_{0}}=\frac{16 \kappa}{9 N^{2}} \Omega_{0}^{4 /(3 N)-1} \ln \frac{\Omega_{1}}{\Omega_{0}}>0 .
$$

The unavailable conversion entransy of the system rises with increasing $\Omega_{0}$.

Spontaneous heat transfer will occur between the subsystems in Figure 2 if they are not in equilibrium. Before the ideal plate is removed, the available conversion entransies of the subsystems are

$$
G_{\mathrm{a}-\mathrm{conv}-\mathrm{s} 1}=\kappa_{\mathrm{s}} \Omega_{\mathrm{s} 1}^{4 /\left(3 N_{\mathrm{s}}\right)}-\kappa_{\mathrm{s}} \Omega_{\mathrm{s} 0}^{4 /\left(3 N_{\mathrm{s}}\right)}-\frac{4 \kappa_{\mathrm{s}}}{3 N_{\mathrm{s}}} \Omega_{\mathrm{s} 0}^{4 /\left(3 N_{\mathrm{s}}\right)} \ln \frac{\Omega_{\mathrm{s} 1}}{\Omega_{\mathrm{s} 0}},
$$

and

$$
G_{\mathrm{a}-\text { conv }-\mathrm{s} 2}=\kappa_{\mathrm{s}} \Omega_{\mathrm{s} 2}^{4 /\left(3 N_{\mathrm{s}}\right)}-\kappa_{\mathrm{s}} \Omega_{\mathrm{s} 0}^{4 /\left(3 N_{\mathrm{s}}\right)}-\frac{4 \kappa_{\mathrm{s}}}{3 N_{\mathrm{s}}} \Omega_{\mathrm{s} 0}^{4 /\left(3 N_{\mathrm{s}}\right)} \ln \frac{\Omega_{\mathrm{s} 2}}{\Omega_{\mathrm{s} 0}},
$$

respectively. The sum of eqs. (41) and (42) is the total available conversion entransy of the system,

$$
\begin{aligned}
G_{\mathrm{a}-\mathrm{conv}}= & \kappa_{\mathrm{s}}\left[\Omega_{\mathrm{s} 1}^{4 /\left(3 N_{\mathrm{s}}\right)}+\Omega_{\mathrm{s} 2}^{4 /\left(3 N_{\mathrm{s}}\right)}\right]-2 \kappa_{\mathrm{s}} \Omega_{\mathrm{s} 0}^{4 /\left(3 N_{\mathrm{s}}\right)} \\
& -\frac{4 \kappa_{\mathrm{s}}}{3 N_{\mathrm{s}}} \Omega_{\mathrm{s} 0}^{4 /\left(3 N_{\mathrm{s}}\right)} \ln \frac{\Omega_{\mathrm{s} 1} \Omega_{\mathrm{s} 2}}{\Omega_{\mathrm{s} 0}^{2}} .
\end{aligned}
$$

Correspondingly, the unavailable conversion entransy is

$$
G_{\text {ua-conv }}=2 \kappa_{\mathrm{s}} \Omega_{\mathrm{s} 0}^{4 /\left(3 N_{\mathrm{s}}\right)}+\frac{4 \kappa_{\mathrm{s}}}{3 N_{\mathrm{s}}} \Omega_{\mathrm{s} 0}^{4 /\left(3 N_{\mathrm{s}}\right)} \ln \frac{\Omega_{\mathrm{s} 1} \Omega_{\mathrm{s} 2}}{\Omega_{\mathrm{s} 0}^{2}} .
$$

The subsystems will finally reach equilibrium after the ideal plate is removed due to the spontaneous heat transfer. The available conversion entransy at equilibrium is

$$
G_{\mathrm{a}-\mathrm{conv}-\mathrm{eq}}=\kappa \Omega_{\mathrm{eq}}^{4 /(3 N)}-\kappa \Omega_{0}^{4 /(3 N)}-\frac{4 \kappa}{3 N} \Omega_{0}^{4 /(3 N)} \ln \frac{\Omega_{\mathrm{eq}}}{\Omega_{0}} .
$$

The unavailable conversion entransy is

$$
G_{\text {ua-conv-eq }}=\kappa \Omega_{0}^{4 /(3 N)}+\frac{4 \kappa}{3 N} \Omega_{0}^{4 /(3 N)} \ln \frac{\Omega_{\text {eq }}}{\Omega_{0}} .
$$

Considering that $N=2 N_{\mathrm{S}}$ and eqs. (19)-(21) and (25), we get

$$
G_{\text {ua-conv }}<G_{\text {ua-conv-eq }},
$$

that is, the spontaneous heat transfer increases both the microstate number of the system and the unavailable conversion entransy.

The comparison between eqs. (43) and (45) with the consideration of eqs. (20), (21), (24) and (47) shows that the second terms on the right side of eqs. (43) and (45) equal each other, the first term on the right side of eq. (43) is bigger than that of eq. (45), and the last term on the right side of eq. (43) is bigger than that of eq. (45). Therefore, it must be true that

$$
G_{\text {a-conv }}>G_{\text {a-conv-eq }} .
$$

The microstate number of the system increases during the spontaneous heat transfer while the available conversion entransy decreases.

In summary, both the available conversion entransy and the unavailable conversion entransy increase with the growth of the initial microstate number when the system is at equilibrium. Raising the temperature of the cold source will increase the microstate number of the system at the cold source temperature and the unavailable conversion entransy, while decrease the available conversion entransy. Spontaneous heat transfer will increase the microstate number of the system and the unavailable conversion entransy, but decrease the available conversion entransy.

\section{Discussion}

As a comparison, we analyze the variations of entropy and available energy in this section after having discussed the variations of the available transport entransy, the unavailable transport entransy, the available conversion entransy and the unavailable conversion entransy with the microstate number. 
Eq.(3) indicates that increasing the initial microstate number will increase the system entropy if the system is at equilibrium. On the other hand, the spontaneous heat transfer in a nonequilibrium state will enlarge the microstate number and the entropy in turn. However, the mechanisms of the entropy increases are different from each other, which is why the variations of the available transport entransy and the available conversion entransy are not consistent. For the equilibrium case, the increase of the microstate number results from the input energy from outside that raises the system temperature. Therefore, both the available transport entransy and the available conversion entransy increase. In the nonequilibrium case, the heat transfer in the system produces entropy generation and increases the microstate number, resulting in the loss of the ability of transporting heat or doing work. Therefore, both the available transport entransy and the available conversion entransy decrease.

At thermal equilibrium, the available energy is

$$
\delta E_{\mathrm{a}-\mathrm{conv}}=\left(1-\frac{T_{0}}{T}\right) \delta Q=\mathrm{d} U-\frac{T_{0}}{T} \mathrm{~d} U,
$$

where $\delta Q$ is the energy transported out of the system. Integrating eq. (49) leads to the available energy of the system,

$$
E_{\text {a-conv }}=\int_{T_{0}}^{T}\left(\mathrm{~d} U-\frac{T_{0}}{T} \mathrm{~d} U\right)=U-U_{0}-U_{0} \ln \frac{T}{T_{0}} .
$$

The derivation of eq. (50) with respect to the system temperature $T$ is always positive, while that with respect to the cold source temperature $T_{0}$ is always negative. Therefore, the available energy increases with the increase in $T$, while it decreases with the increase in $T_{0}$. By considering the relationship between the temperature and the microstate number, one finds that the available energy increases when energy is transported into the system and the microstate number of the system increases, while the available energy decreases with increasing the microstate number of the system at the temperature of the cold source.

If the system is not at thermal equilibrium, we can also discuss the relation between the available energy and the microstate number. The total available energy of the two subsystems in Figure 2 is

$$
\begin{aligned}
E_{\mathrm{a}-\mathrm{conv}} & =E_{\mathrm{a}-\mathrm{conv}-\mathrm{s} 1}+E_{\mathrm{a}-\mathrm{conv}-\mathrm{s} 2} \\
& =\left(U_{1}+U_{2}\right)-\left(U_{10}+U_{20}\right)-U_{10} \ln \frac{T_{\mathrm{s} 1}}{T_{0}}-U_{20} \ln \frac{T_{\mathrm{s} 2}}{T_{0}} \\
& =U-U_{0}-U_{10} \ln \frac{T_{\mathrm{s} 1}}{T_{0}}-U_{20} \ln \frac{T_{\mathrm{s} 2}}{T_{0}} \\
& =U-U_{0}-U_{0}\left(\ln \sqrt{T_{\mathrm{s} 1} T_{\mathrm{s} 2}}-\ln T_{0}\right),
\end{aligned}
$$

where $U_{1}$ and $U_{2}$ are the initial internal energies subsystems 1 and $2, U_{10}$ and $U_{20}$ are the internal energies of the subsystems at the cold source temperature (which are equal). When the system reaches thermal equilibrium,

$$
\begin{aligned}
& E_{\text {a-conv-eq }}=U-U_{0}-U_{0} \ln \frac{T_{\text {eq }}}{T_{0}} \\
& =U-U_{0}-U_{0}\left(\ln T_{\text {eq }}-\ln T_{0}\right) .
\end{aligned}
$$

Combining eqs. (51) and (52) leads to

$$
\begin{aligned}
E_{\mathrm{a}-\mathrm{conv}}-E_{\mathrm{a}-\mathrm{conv}-\mathrm{eq}} & =-U_{0}\left(\ln \sqrt{T_{\mathrm{s} 1} T_{\mathrm{s} 2}}-\ln T_{\mathrm{eq}}\right) \\
& =-U_{0}\left(\ln \sqrt{T_{\mathrm{s} 1} T_{\mathrm{s} 2}}-\ln \frac{T_{\mathrm{s} 1}+T_{\mathrm{s} 2}}{2}\right)>0 .
\end{aligned}
$$

It is apparent that the available energy of the system decreases during the spontaneous heat transfer. The available energy decreases with increasing the microstate number in this case because the microstate number increases during the heat transfer.

The analyses in this section show that the variation of the available energy with the microstate number is not consistent with that of the entropy, but is consistent with that of the available conversion entransy. Therefore, the concept of the available conversion entransy can describe the change direction of the available energy in the cases discussed in this paper.

\section{Conclusions}

We have investigated the variations of the available transport entransy, the unavailable transport entransy, the available conversion entransy and the unavailable conversion entransy with the microstate number for a system. Some conclusions are:

(1) The unavailable transport entransy only depends on the microstate number of the system at the cold source temperature during the heat transfer between the system and the cold source. Bigger $\Omega_{0}$ leads to a larger unavailable transport entransy and a smaller available transport entransy. The available transport entransy is determined not only by $\Omega_{0}$ but also by the initial microstate number of the system $\Omega_{1}$. The available transport entransy increases with $\Omega_{1}$ if the system is in equilibrium, and the increase of $\Omega_{1}$ results from the energy input to the system. On the other hand, if the system is not at equilibrium and the increase of $\Omega_{1}$ results from the spontaneous heat transfer, the available transport entransy will decrease with increasing $\Omega_{1}$.

(2) Both the available conversion entransy and the unavailable conversion entransy for an equilibrium system will rise with increasing $\Omega_{1}$ caused by energy input to the system. Raising the temperature of the cold source increases both the microstate number of the system at the cold source temperature and the unavailable conversion entransy, but decreases the available conversion entransy. The spontaneous heat transfer at nonequilibrium increases the microstate number of the system, increases the unavailable conversion entransy but reduces the available conversion entransy. 
(3) The variation of the available energy with the microstate number is consistent with that of the available conversion entransy. The concept of available conversion entransy can describe the change direction of the available energy in the cases discussed in this paper.

This work was supported by the National Natural Science Foundation of China (51106082) and the Tsinghua University Initiative Scientific Research Program.

1 Zhao K H, Luo W Y. Thermotics (in Chinese). Beijing: Higher Education Press, 2002

2 Feng D, Feng S T. The World of Entropy (in Chinese). Beijing: Science Press, 2008. 104-160

3 Ge Y L, Chen L G, Sun F R. Optimal paths of piston motion of irreversible Otto cycle heat engines for minimum entropy generation. Sci China-Phys Mech Astron, 2010, 40: 1115-1129

4 Guo Z Y, Zhu H Y, Liang X G. Entransy-A physical quantity describing heat transfer ability. Int J Heat Mass Transfer, 2007, 50: 2545-2556

5 Chen L G, Wei S H, Sun F R. Constructal entransy dissipation minimization for 'volume-point' heat conduction. J Phys D: Appl Phys, 2008, 41: 195506

6 Cheng X T, Xu X H, Liang X G. Homogenization of temperature field and temperature gradient field. Sci China Ser E-Tech Sci, 2009, 52: 2937-2942

7 Xie Z H, Chen L G, Sun F R. Constructal optimization for geometry of cavity by taking entransy dissipation minimization as objective. Sci China Ser E-Tech Sci, 2009, 52: 3413-3504

8 Xie Z H, Chen L G, Sun F R. Constructal optimization on T-shaped cavity based on entransy dissipation minimization. Chin Sci Bull, 2009, 54: 4418-4427

9 Wei S H, Chen L G, Sun F R. Constructal optimization of discrete and continuous-variable cross-section conducting path based on entransy dissipation rate minimization. Sci China: Tech Sci, 2010, 53: $1666-1677$

10 Wei S H, Chen L G, Sun F R. Constructal entransy dissipation minimization for "volume-point" heat conduction without the premise of optimized last-order construct. Int J Exergy, 2010, 7: 627-639

11 Xiao Q H, Chen L G, Sun F R. Constructal entransy dissipation rate minimization for "disc-to-point" heat conduction. Chin Sci Bull, 2011, 56: 102-112

12 Xiao Q H, Chen L G, Sun F R. Constructal entransy dissipation rate minimization for heat conduction based on a tapered element. Chin Sci Bull, 2011, 56: 2400-2410

13 Chen L, Wei S, Sun F. Constructal entransy dissipation rate minimization of a disc. Int J Heat Mass Transfer, 2011, 54: 210-216
14 Chen Q, Ren J, Guo Z. Field synergy analysis and optimization of decontamination ventilation designs. Int J Heat Mass Tranfer, 2008, 51: 873-881

15 Chen Q, Ren J X. Generalized thermal resistance for convective heat transfer and its relation to entransy dissipation. Chin Sci Bull, 2008, 53: 3753-3761

16 Chen Q, Wang M R, Pan N, et al. Optimization principles for convective heat transfer. Energy, 2009, 34: 1199-1206

17 Liu W, Liu Z, Jia H, et al. Entransy expression of the second law of thermodynamics and its application to optimization in heat transfer process. Int J Heat Mass Transfer, 2011, 54: 3049-3059

18 Wu J, Liang X G. Application of entransy dissipation extremum principle in radiative heat transfer optimization. Sci China Ser E-Tech Sci, 2008, 51: 1306-1314

19 Cheng X T, Liang X G. Entransy flux of thermal radiation and its application to enclosures with opaque surfaces. Int J Heat Mass Transfer, 2011, 54: 269-278

20 Cheng X T, Xu X H, Liang X G. Radiative entransy flux in enclosures with non-isothermal or non-grey, opaque, diffuse surfaces and its application. Sci China: Tech Sci, 2011, 54: 2446-2456

21 Liu X B, Meng J A, Guo Z Y. Thermal resistance analysis for the heat exchangers based on entransy dissipation. Prog Nat Sci, 2008, 18: $1186-1190$

22 Liu X B, Meng J A, Guo Z Y. Entropy generation extremum and entransy dissipation extremum for heat exchanger optimization. Chin Sci Bull, 2009, 54: 943-947

23 Liu X B, Guo Z Y. A novel method for heat exchanger analysis. Acta Phys Sin, 2009, 58: 4766-4771

24 Wei S, Chen L, Sun F. Constructal entransy dissipation rate minimization of round tube heat exchanger cross-section. Int J Therm Sci, 2011, 50: 1285-1292

25 Xia S J, Chen L G, Sun F R. Optimization for entransy dissipation minimization in heat exchanger. Chin Sci Bull, 2009, 54: 3572-3578

26 Guo J F, Xu M T, Cheng L. Principle of equipartition of entransy dissipation for heat exchanger design. Sci China Tech Sci, 2010, 53: 1309-1314

27 Guo Z Y, Liu X B, Tao W Q, et al. Effectiveness-thermal resistance method for heat exchanger design and analysis. Int J Heat Mass Transfer, 2010, 53: 2877-2884

28 Xia S, Chen L, Sun F. Entransy dissipation minimization for liquid-solid phase processes. Sci China Tech Sci, 2010, 53: 960-968

29 Zhu W H, Gu Y Q. Fundamentals of Statistical Physics (in Chinese). Beijing: Tsinghua University Press, 1983. 153-194

30 Cheng X T, Liang X G, Xu X H. Microscopic expression of entransy. Acta Phys Sin, 2011, 60: 060512

31 Wu J. Potential energy (Entransy) in thermal science and its application (in Chinese). Dissertation for Doctoral Degree. Beijing: Tsinghua University, 2009. 72-106

32 Hu G J, Guo Z Y. Entransy and entropy revisited. Chin Sci Bull, 2011, 56: 2974-2977

Open Access This article is distributed under the terms of the Creative Commons Attribution License which permits any use, distribution, and reproduction in any medium, provided the original author(s) and source are credited. 